\title{
Hyperglycemia in pasireotide-treated patients with acromegaly and its treatment
}

\author{
Anton Luger ${ }^{1}$
}

Received: 3 June 2016 / Accepted: 18 June 2016 / Published online: 8 July 2016

(C) Springer Science+Business Media New York 2016

M.A. Schmidt et al. analyzed data from the previously published PAOLA study [1], a phase 3 study where the safety and efficacy of pasireotide LAR was compared to the two first-generation somatostatin analogs (SSA) octreotide and lanreotide in acromegalic patients not controlled with either of these two drugs, with special attention to the effect of pasireotide on glucose homeostasis [2].

First, it should be mentioned that the patients of the PAOLA study represent a selected population with a highly increased risk of developing hyperglycemia or diabetes due to the duration, severity, and previous therapy of the growth hormone (GH) excess that could not be normalized by prior interventions (maximal doses of first-generation SSA \pm prior surgery). Already at study entry approximately $30 \%$ of the patients were receiving antidiabetic treatment and another $20 \%$ had fasting glucose concentrations above $100 \mathrm{mg} / \mathrm{dL}$ and thus must be classified as having prediabetes with its known increased risk of developing diabetes. Pasireotide LAR could be seen as trigger in diabetes-prone subjects. This is further supported by the observation that $52-71 \%$ of the patients with fasting glucose concentrations above $100 \mathrm{mg} / \mathrm{dL}$ developed hyperglycemia during pasireotide LAR treatment.

The observation that glycated hemoglobin ( $\mathrm{HbAlc}$ ) increased during the first 2-3 months and plataued thereafter should be interpreted with caution. Whereas this can be interpreted as precipitation or worsening of glucose

Anton Luger

anton.luger@meduniwien.ac.at

1 Division of Endocrinology and Metabolism, Department of Medicine III, Medical University and General Hospital of Vienna, Waehringer Guertel 18-20, 1090 Vienna, Austria metabolism shortly after starting therapy with pasireotide in patients already at risk and no further effect thereafter, this might also partly be due to initiation of antidiabetic treatment and/or dose adaptation of glucose-lowering drugs in patients under close medical observation in the setting of a clinical trial. In addition, improved control of GH excess is expected to result in better glycemic control [3], thereby counteracting the diabetogenic effect of pasireotide.

Pasireotide has a higher affinity to somatostatin receptor subtype (SSTR) 5 and a slightly lower affinity to SSTR2 compared to octreotide and lanreotide [4]. The different expression of SSTR2 and SSTR5 on pancreatic $\alpha$ and $\beta$ cells, respectively [5], is presumed to result in greater suppression of insulin secretion and lesser decrease of glucagon with pasireotide when compared with the firstgeneration SSAs. Indeed, potent suppression of insulin along with suppression of glucagon like peptide-1 (GLP-1), glucose-dependent insulinotropic polypeptide (GIP), and glucagon secretion has been demonstrated in healthy volunteers receiving pasireotide, whereas peripheral as well as hepatic insulin sensitivity were not affected [6]. Thus, if hyperglycemia in pasireotide-treated patients with acromegaly is precipitated at least in part by suppression of insulin and GLP-1 it appears logical that pharmacotherapy targeting insulin release and mimicking incretin action should be the treatment of choice. In fact, superiority of the dipeptidyl peptidase-4 (DPP-4) inhibitor vildagliptin and the GLP-1 agonist liraglutide over the insulinotropic drug nateglinide and metformin has been demonstrated, albeit only in a study in healthy controls that might differ from patients with acromegaly treated with pasireotide [7]. The superiority of GLP-1 agonists over DPP-4 inhibitors is anticipated not only due to the known greater potency of GLP-1 agonists, but also due to their different mechanism of action since 
inhibition of GLP-1 degradation by DPP-4 inhibitors sets in at lower GLP-1 concentrations when they already have been suppressed by pasireotide. In any case metformin should be part of the antidiabetic treatment in insulin-resistant acromegalic patients even if it had no impressive effect when given as monotherapy in the above-mentioned trial in normal controls with normal insulin sensitivity. The same line of argument might apply for other antidiabetic drugs targeting insulin resistance such as glitazones but their efficacy remains to be proven also. Evidently, insulin is the treatment option in patients with insufficient response to oral antidiabetic drugs. Finally, also substances of the last class of antidiabetic drugs that entered the market, the SGLT2 inhibitors, can be expected to positively affect pasireotideinduced hyperglycemia but no evidence is available yet.

A different treatment option would be the combination of SSA with pegvisomant with its previously reported beneficial effects on glucose metabolism [8]. Thereby the positive effects of both drugs could be exploited and possible side effects minimized by reduction of the dose of both substances. Surprisingly, hitherto published studies do not support this consideration impressively [9, 10]. Nevertheless, in acromegalic patients with pre-existing diabetes mellitus treatment with pegvisomant alone or in combination with pasireotide should be considered.

Taken together, pasireotide LAR is a potent new treatment option in acromegaly associated with more severe impairment of glucose metabolism than first-generation SSA. These side effects need to be balanced against the deleterious effects of uncontrolled GH excess. In patients starting pasireotide LAR treatment fasting glucose concentrations and HbA1c should be monitored carefully, especially in the initial period. The clinical experience with pasireotide LAR in accordance with the analysis of the data from the PAOLA study reported in this issue of Endocrine show that glucose metabolism stabilizes after an initial worsening in the first few weeks, sometimes even without pharmacological intervention. The underlying mechanisms of this phenomenon remain to be identified.

Treatment options of pasireotide LAR-exacerbated derangements of glucose homeostasis in acromegalic patients should aim primarily to counteract the underlying pathomechanisms, i.e., stimulate insulin and GLP-1 secretion and action, decrease glucagon secretion and decrease insulin resistance. Metformin is a logical candidate combining increase in insulin sensitivity with additional action on DPP-4 activity, thereby increasing GLP-1 concentrations followed by enhanced insulin and diminished glucagon secretion. Arguments for the use of nearly all classes of antidiabetic drugs can be found, until present, however, these are mainly theoretical considerations not yet supported by hard facts and awaiting proof by studies designed to specifically investigate the effects of the many antidiabetic drugs available for the treatment of diabetes. A similar conclusion has been formulated in a recent guideline on the treatment of hyperglycemia in acromegaly by the Italian Society for the Study of Diabetes and Endocrinological Society [11].

\section{Compliance with ethical standards}

Conflicts of interest The author has received fees for lectures and/or consulting from Ipsen, Novartis, Pfizer, Novo Nordisk, Eli Lilly, Sanofi, Boehringer Ingelheim, Astra Zeneca, MSD, and Takeda.

\section{References}

1 M.R. Gadelha, M.D. Bronstein, T. Brue et al., Pasireotide versus continued treatment with octreotide or lanreotide in patients with inadequately controlled acromegaly (PAOLA): a randomised, phase 3 trial. Lancet Diabetes Endocrinol. 2, 875-884 (2014)

2 H.A. Schmid, T. Brue, A.M. Colao et al., Effect of pasireotide on glucose- and growth hormone-related biomarkers in patients with inadequately controlled acromegaly. Endocrine (2016). doi:10.1007/s12020-016-0895-8

3 M. Tzanela, D.A. Vassiliadi, N. Gavalas et al., Glucose homeostasis in patients with acromegaly treated with surgery or somatostatin analogues. Clin. Endocrinol. 75, 96-102 (2011)

4 C. Bruns, I. Lewis, U. Briner et al., SOM230: a novel somatostatin peptidomimetic with broad somatotropin release inhibiting factor (SRIF) receptor binding and a unique antisecretory profile. Eur. J. Endocrinol. 146, 707-716 (2002)

5 G. Weckbecker, I. Lewis, R. Albert et al., Opportunities in somatostatin research: biological, chemical and therapeutic aspects. Nature Rev. Drug Discov. 2, 999-1017 (2003)

6 R.R. Henry, T.P. Ciaraldi, D. Armstrong et al., Hyperglycemia associated with pasireotide: results from a mechanistic study in healthy volunteers. J. Clin. Endocrinol. Metab. 98, 3446-3453 (2013)

7 A. Breitschaft, K. Hu, K. Hermosillo Resendiz et al., Management of hyperglycemia associated with pasireotide (SOM230): healthy volunteer study. Diabetes Res. Clin. Pract. 103, 458-465 (2014)

8 A.L. Barkan, P. Burman, D.R. Clemmons et al., Glucose homeostasis and safety in patients with acromegaly converted from long-acting octreotide to pegvisomant. J. Clin. Endocrinol. Metab. 90, 5684-5691 (2005)

9 L. de Marinis, A. Bianchi, A. Fusco et al., Long-term effects of the combination of pegvisomant with somatostatin analogs (SSA) on glucose homeostasis in non-diabetic patients with active acromegaly partially resistant to SSA. Pituitary 10, 227-232 (2007)

10 M. Madsen, P.L. Poulsen, H. Orskov et al., Cotreatment with pegvisomant and a somatostatin analog (SA) in SA-responsive acromegalic patients. J. Clin. Endocrinol. Metab. 96, 2405-2413 (2011)

11 M.G. Baroni, F. Giorgino, V. Pezzino et al., Italian Society for the Study of Diabetes (SID)/Italian Endocrinological Society (SIE) guidelines on the treatment of hyperglycemia in Cushing's syndrome and acromegaly. J. Endocrinol. Invest. 39, 235-255 (2016) 